\title{
Jahresbericht 2010
}

Korrespondenz:

Dr. med. U. Leibundgut Präsident der Stiftungskommission Lange Gasse 78 CH-4052 Basel
Die Hilfskasse für Schweizer Ärzte unterstützte während des vergangenen Jahres 26 (2009: 31) ohne eigene Schuld in materielle Not geratene Ärztinnen und Ärzte sowie Angehörige und Nachkommen von verstorbenen Kolleginnen und Kollegen.

10 Unterstützungen fielen im Laufe des Jahres weg. Neu hinzu kamen im Jahr 2010 fünf Unterstützungen.

Auf der Ertragsseite stehen Einnahmen aus Spenden von 121660 CHF (Durchschnitt der letzten 10 Jahre: 155979 CHF) sowie Zinsen und Dividenden des Stiftungskapitals von 76530 CHF.

Beim Aufwand haben wir Ausgaben für Unterstützungen von 235250 CHF (Durchschnitt der letzten 10 Jahre: 312368 CHF). Die Verwaltungsausgaben beliefen sich auf 44312 CHF.

Die Differenz zwischen Einnahmen aus Spenden sowie Zins- und Dividendenertrag aus Kapital und den Aufwendungen konnte in gutgehenden Börsenjahren durch Realisierung von Kursgewinnen an der Börse wettgemacht werden. Im vergangenen Jahr konnte lediglich ein Kursgewinn von 18931 CHF realisiert werden. Diese ungünstige Entwicklung führte dazu, dass das Stiftungsvermögen auf 3027948 CHF (2009:3 092600 CHF) abgenommen hat.

Das Stiftungsvermögen ist in einem Mix aus Obligationen und Aktien angelegt, der den Vorschriften für die berufliche Vorsorge BVG entspricht. Erfolgsrechnung und Bilanz sowie Verwaltung des Stiftungsvermögens werden jährlich durch die Stiftungsaufsichtsbehörde des Justizdepartementes Kanton Basel-Stadt überprüft.
Spendenbeträge unter $500 \mathrm{CHF}$, die wir zur Minimierung unserer Verwaltungskosten nicht einzeln verdanken können, bilden den traditionellen Grundstock unserer Stiftung; diese seien deshalb an dieser Stelle ganz herzlich verdankt.

Die Jahresrechnung der Hilfskasse wurde von der Firma Axt Treuhand revidiert und für in Ordnung befunden. Für ihre Arbeit sei herzlich gedankt. Einzelheiten zur Jahresrechnung können der nachfolgenden Bilanz und Erfolgsrechnung entnommen werden.

Ich danke allen Kommissionsmitgliedern für ihre Arbeit, die sie im vergangenen Jahr ehrenamtlich geleistet haben. Mein Dank geht auch an die Herren J. Kobler und W. Mahrer von der Bank La Roche \& Co für die Buchhaltung und Vermögensverwaltung.

\section{Die Stiftungskommission der Hilfskasse} für Schweizer Ärzte:

Dr. U. Leibundgut, Präsident

Frau Dr. S. Stöhr

Dr. U. Lüscher, Kassier

PC-Konto der Hilfskasse für Schweizer Ärzte:

40-644-3 Basel 


\section{Jahresrechnung 2010 (in Franken)}

Bilanz per 31. Dezember 2010

Konti Bank La Roche

Konto Postcheck

Debitor Verrechnungssteuer

Wertschriften

Passive Rechnungsabgrenzung

Rückstellungen auf Wertschriften

Vermögen per 31. Dezember 2010

Dr. J. Ambühl-Stiftung

Dr. J. Genhard-Stiftung

Dr. J. B. Wutz-Stiftung

Hilfskasse per 1. Januar

Vermögensabnahme

Erfolgsrechnung 2010

Spenden, Zeugnisgelder

Kapitalertrag

Real. Kursgewinne auf Wertschriften

Unterstützungen

Bankspesen und Verwaltungsgebühren

Kursverluste FW-Konti

Vermögensabnahme
Aktiven

Passiven

117843.68

109051.47

19978.75

2781074.70

19307.45

852000.00

90000.00

20000.00

19450.75

2091165.80

2220616.55

$-63975.40$

3027948.60

3027948.60

Aufwand

Ertrag

121660.30

76530.00

18931.85

235250.00

44312.75

1534.80

$-63975.40$

217122.15 